\section{Fetal, neonatal, and post-neonatal mortality in the 2015 Pelotas (Brazil) birth cohort and associated factors}

\author{
Mortalidade fetal, neonatal e pós-neonatal e fatores \\ associados na coorte de nascimentos de 2015 de \\ Pelotas, Rio Grande do Sul, Brasil
}

\author{
Mortalidad fetal, neonatal y postneonatal en la \\ cohorte de nacimientos en Pelotas, Rio Grande \\ do Sul, Brasil, 2015, y sus factores asociados
}

Andrea Ramirez Varela 1

Bruna Celestino Schneider 1

Susana Bubach 1,2

Mariangela Freitas Silveira 1

Andréa Dâmaso Bertoldi 1

Lina Sofia Moron Duarte 1

Ana Maria B. Menezes 1

Marlos R. Domingues 1

Diego G. Bassani 1

\begin{abstract}
This study aimed to describe fetal, neonatal, and post-neonatal mortality and associated factors in participants of the 2015 Pelotas (Brazil) birth cohort. The child mortality sub-study followed up all deaths in the first year of life. Data were collected on intrauterine fetal deaths (weight $\geq 500 \mathrm{~g}$ and/or gestational age $\geq 20$ weeks), neonatal deaths ( $<28$ days of life), and post-neonatal deaths (from 28 days to the end of the first year of life). Descriptive analyses using the Pearson chi-square test and a multinomial logistic regression to estimate the risk of fetal, neonatal, and post-neonatal deaths compared to live infants in the cohort (reference group) were performed. Data from 4,329 eligible births were collected, of which 54 died during the fetal period. Of the 4,275 eligible live births, 59 died in the first year of life. An association between fetal, neonatal, and post-neonatal deaths $(O R=15.60,7.63$, and 5.51 respectively) was found, as well as less than six prenatal consultations. Compared to live infants, fetal deaths were more likely to occur in non-white mothers, and neonatal deaths were 14.09 times more likely to occur in a preterm gestational age ( $<37$ weeks). Compared to live infants, infants that were born in a C-section delivery had 3.71 increased odds of post-neonatal death. Additionally, neonatal deaths were 102.37 times more likely to have a low Apgar score on the fifth minute after birth. These findings show the need for early interventions during pregnancy, ensuring access to adequate prenatal care.
\end{abstract}

Infant Mortality; Cohort Studies; Risk Factors

\author{
Correspondence \\ S. Bubach \\ Universidade Federal do Espirito Santo. \\ Rodovia BR-101 Norte, km 60, São Mateus, ES \\ 29932-540, Brasil. \\ sbubach@gmail.com \\ 1 Programa de Pós-graduação em Epidemiologia, Universidade \\ Federal de Pelotas, Pelotas, Brasil. \\ 2 Universidade Federal do Espírito Santo, São Matheus, Brasil.
}




\section{Background}

Worldwide infant mortality has declined over the past 25 years, but it still represents a public health priority, since, in 2016, 75\% of all deaths in children under five occurred in the first year of life 1. Inequalities between mortality rates in the first year of life in different regions of the world still persist, and the current risk of death before reaching the age of one in Latin America is twice the risk for children born in North America 1.

In Brazil, in recent decades, there has been a reduction in infant mortality rates (IMR) from 53.4 per 1,000 live births in 1990 to about 13.5 per 1,000 live births in $2016^{2}$. This decrease can be explained by the implementation of a series of policies, programs, and improvements in social, sanitary, and health conditions, such as poverty and fertility reduction, improvement in female education 3 , urbanization expansion, Brazilian Income Transfer Program (2004), and the increased access to clean water and sanitation that started about 80 years ago 4,5. Additionally, national programs such as the National Vaccination Program (1973), National Health Assistance for Women and Children (1984), Brazilian Unified National Health System (SUS, 1988), Family Health Strategy (1994), National Humanization Program for Birth Attention (2000), and Healthcare Network for Mothers and Children (2011), among others, contributed to improvements in maternal and infant health services 4 .

However, despite a 2/3 decrease in infant mortality over the 1990-2015 period, which lead Brazil to meet four of the millennium development goals 4 , continuous monitoring of infant mortality (children under one year) remains a priority in the country. For example, even though the neonatal mortality rate decreased from 23.1 to 9.5 per 1,000 live births (1990-2015), neonatal deaths represented $70 \%$ of total deaths during childhood in 2015 . Additionally, infant deaths in 2015 had preventable 6 infectious, nutritional, maternal, and neonatal causes 5 (e.g., a rise in caesarean sections; increased congenital syphilis, premature births, and low birth weight 4 ), all related to poor mother-child health 7 . Previous studies have shown that data collection, processing, and analysis of factors associated with infant morbidity and mortality had supported an adequate allocation of resources for planning and restructuring the network of maternal and child care services with the ultimate goal of reducing hospitalizations and infant deaths 7,8,9. These data have been collected through information systems and epidemiologic studies, which are fundamental and of great relevance for the country. Specific limitations of information systems are related to sub-registries, particularly in regions with a historic lack of infant death information 4,10,11,12,13,14. For example, in 2012 a Brazilian study actively searched for infant deaths in 79 small and medium size municipalities in the North and Northeast of the country, and found that public health monitoring coverage was below $80 \%$ in seven states 10 . Additionally, the lack of information on the mandatory reporting forms and lack of communication between live and death births databases and information systems limit a complex analyses of these indicators 12,13,14. For example, low birth weight has been reported as more prevalent in the South and Southeast regions of the country and less prevalent in the North and Northeast regions of the country 15. However, this represents a mismatch when analysing regional sociodemographic patterns, as it is known that the South and Southeast regions do not have most of their population matching the cases that have been described as having low maternal education and black and brown skin colors 4 .

Epidemiologic studies on maternal and child health also contribute to providing quality data; assessing causes of death, hospitalizations, factors associated with survival, gaps in the health care network; and providing possible solutions for maternal and infant health problems. Primary data broadens, details, and allows one to associate maternal variables with infant mortality and morbidity outcomes, which is of particular importance in Brazil, where $41 \%$ of infant deaths have been reported to occur during the first six days of life and are closely related to prenatal and birth periods 5 .

The 2015 Pelotas (Brazil) birth cohort 16 is the fourth edition of the series of Pelotas (Brazil) birth cohort studies, preceded by cohorts initiated in 1982 17, 1993 18, and 2004 19. The original study began with the follow-up of participants during prenatal care, between May 2014 and December 2015, to identify pregnant women expected to give birth during 2015. Subsequently, the children were documented immediately after birth and at 3, 12, and 24 months of age. Infant morbidity and mortality were monitored in the first year of life in the morbi-mortality sub-study. This study aimed to describe fetal, neonatal, and post-neonatal mortality and associated factors in participants of the 2015 Pelotas (Brazil) birth cohort. 


\section{Methods}

The 2015 Pelotas (Brazil) birth cohort mortality sub-study recorded all deaths (fetal and infant) of children born in the cohort during the first year of life, corresponding to the period between January 1, 2015 and December 31, 2016. The flowchart of the 2015 Pelotas birth cohort participants is shown in the Supplementary Material (Supplementary Figure S1: http://cadernos.ensp.fiocruz.br/site/pub lic_site/arquivo/supl-e00072918_2195.pdf).

Before beginning data collection and to obtain access authorizations, the following registration services, deaths and hospitalization investigation sites in the municipality were contacted: (1) Death Registry Office of the Municipal Health Department (SMS); (2) Child Health Program (PSC)/Epidemiological Surveillance; (3) Regional Health Superintendence/Death Investigation Committee; and (4) hospitals with maternity wards and beds for pediatric hospitalization in Pelotas: São Francisco de Paula University Hospital of the Catholic University of Pelotas (UCPel); University Support Foundation Hospital (FAU) of the Federal University of Pelotas (UFPel); Santa Casa and Miguel Piltcher hospitals.

\section{Instruments}

The outcomes reported in this sub-study referred to fetal/stillbirth loss (intrauterine fetal death, at least $500 \mathrm{~g}$ in weight and/or gestational age of 20 weeks or more); neonatal death ( $<28$ days of life); and post-neonatal/infant death (deaths of live births before completing 12 months of life).

The following data collection tools were developed: (1) fetal, neonatal, and infant mortality, including information on the death certificate; (2) mortality from birth to three months of age, including information according to medical records; (3) mortality between four and 12 months of life, including data from hospital medical records. The Supplementary Material (Supplementary Table S1: http://cadernos.ensp.fiocruz.br/site/public_site/arquivo/supl-e00072918_2195.pdf) presents the synthesis of the information collected and used in the evaluation of neonatal and infant mortality and associated risk factors.

\section{Data collection}

Interviewers visited the city hospitals daily to identify cases of fetal, neonatal, and post-neonatal deaths among cohort participants and maternal and child characteristics related to mortality and morbidity. The sub-study team worked together with the perinatal cohort follow-up team during 2015. After the closure of the perinatal follow-up, in the period between January 1, 2016 and December 31, 2016, lists provided by the hospitals that included data related to hospital visits (hospitalization or emergency care visit records) and lists of deaths provided by the SMS were used.

Additionally, the cohort follow-up teams provided mortality information obtained during follow up visits scheduled to families at their homes. In cases where death or hospital care information (hospitalization or emergency room visit records) had not been collected or were incomplete, the death certificate was requested from the SMS and the medical records were obtained from the hospital by the Medical File and Statistics System (SAME). Information on the list of all births recorded in 2015 from the city Brazilian Information System on Live Births (SINASC) was provided by the SMS and was used to confirm the number of births and to track possible cohort losses. The Supplementary Material (Supplementary Figure S2: http://cadernos.ensp.fiocruz.br/site/public_site/arquivo/suple00072918_2195.pdf) describes the investigation sequence of hospitalizations and deaths of the 2015 Pelotas (Brazil) birth cohort.

The independent variables about maternal characteristics were collected along with the mortality questions: age (years), education (complete years of study), reproductive history (parity, threat of abortion or premature delivery, abortion history, twin pregnancy) and delivery (type, professional who attended, gestational age, professional who attended the delivery is the same that performed the prenatal care), health problems or diseases (infectious and cardiovascular disease, anaemia, depression, or mental illness); and child characteristics: sex, Apgar score in the fifth minute (defined according to the American College of Obstetricians and Gynecologists recommendations) 20,21. Information 
that was not included in death certificates and patient records was obtained from the perinatal cohort follow-up team, such as skin color (self-reporting), marital status, wealth index (Brazilian wealth index classification. Associação Brasileira de Empresas de Pesquisa. http://www.abep.org), maternal health characteristics during pregnancy (tobacco and alcohol use, weight gain, number of prenatal care consultations, infectious and cardiovascular disease, anaemia, depression, or mental illness), and birth weight (measured by the SECA portable paediatric scales model 736).

\section{Supervision, quality control, and data consistency}

Data collection was supervised by a team of field supervisors. Interviewers and supervisors held a weekly supervisory meeting to discuss fieldwork progress and quality control procedures relating to data collection and transfer to the database.

\section{Statistical analyses}

Mortality rates were estimated from standardized international definitions and their respective confidence intervals were estimated 2 . Descriptive analyses of the variables associated with fetal, neonatal, and post-neonatal mortality were performed using the Pearson's chi-squared test. A multinomial logistic regression was conducted to estimate the association between deaths and risk factors. A dependent variable was used with the following categories: fetal death, neonatal death, post-neonatal death, and living infants (reference group). The risk of fetal, neonatal, and post-neonatal deaths and associated factors was estimated using a hierarchical 22 conceptual model with backward elimination of variables (Figure 1). At each level of the analysis model, the variables with a p-value $\leq 0.2$ were retained in the model. In the final model, variables that were statistically associated $(\mathrm{p}<0.05)$ with the outcome were included.

The mortality sub-study of the 2015 Pelotas (Brazil) birth cohort was approved by the Ethics Research Committee (n. 522.064) of the School of Physical Education, UFPel (CAAE $\mathrm{n}$. 26746414.50000.5313). Access to personal information on mortality was obtained after the participants signed the Informed Consent Form.

\section{Results}

The 4,387 eligible births for inclusion in the 2015 Pelotas (Brazil) birth cohort were collected, of which seven mothers were lost and 51 refused to participate with their children in the study. Of the 4,329 eligible births, 54 died in the fetal period. The fetal mortality rate (FMR) was 12.5 stillbirths/1,000 births [95\% confidence interval (95\%CI): 9.1-15.8]. Of the 4,275 eligible live births, 59 died in the first year of life, and the IMR was 13.8/1,000 live births (95\%CI: 10.3-7.3), presented in Table 1.

Concerning sociodemographic characteristics, over $80 \%$ of the cohort mothers were 20 years or older, had studied for six or more years, and reported having a partner. More than 2/3 of these women self-reported white skin color and presented low and medium socioeconomic status (ABEP classes C-D-E). When the general sample of maternal characteristics was compared to those of mothers whose children died, statistically significant differences were observed, since $97 \%$ of the latter had a medium or low socioeconomic status, almost $40 \%$ studied five years or less, and 1/4 did not have a partner.

Regarding the characteristics of the general sample of infants at birth, $82 \%$ were born at term, 90\% had a weight greater than 2,500 grams, and 98.4\% obtained an Apgar score in the 5th minute of life between 7 and 10 points (recognized as an adequate health state of the newborn immediately after birth).

Compared to the live infant/newborn population, neonatal deaths were likely to be preterm (13.3\% compared to $18 \%$ ), were more likely to have low birth weight (54.1\% compared to $10 \%)$ and more likely to have a low Apgar score during the 5 th minute after birth (47.1\% compared to $1.6 \%)$, as presented in Table 2. 


\section{Figure 1}

Hierarchical model of associated factors used in the analysis of infant mortality up to 12 months of age in the 2015 Pelotas (Brazil) birth cohort.

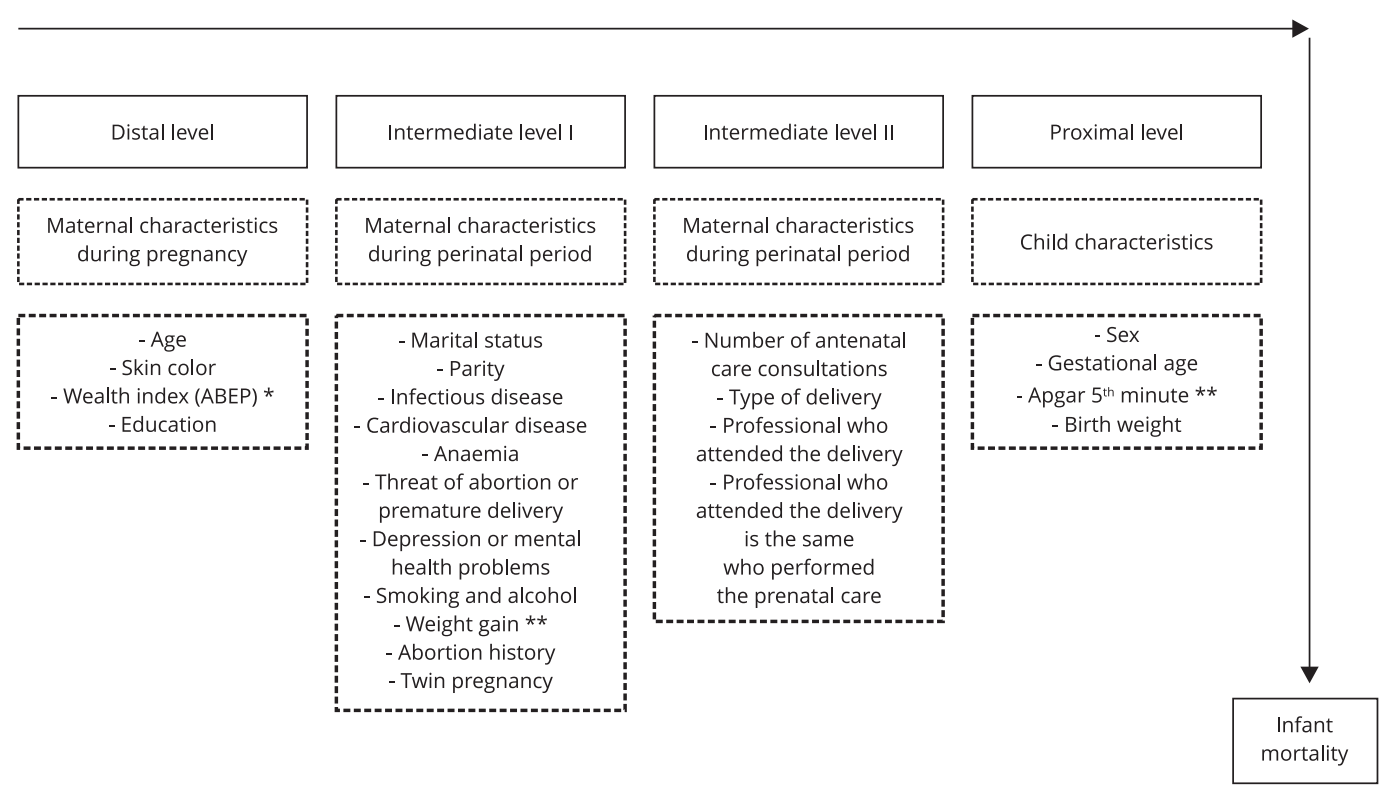

* Brazilian wealth index classification (Associação Brasileira de Empresas de Pesquisa. http://www.abep.org);

** Variables defined according to the American Academy of Pediatrics Committee on Fetus and Newborn, American

College of Obstetricians and Gynecologists Committee on Obstetric Practice recommendations.

Post-neonatal deaths were more likely to be preterm (47.4\% compared to $18 \%$ ), have low birth weight (36.4\% compared to $10 \%)$, and have a low Apgar score in $5^{\text {th }}$ minute after birth (4.6\% compared to $1.6 \%$ ) (Table 2).

Table 3 presents the unadjusted analysis of the risk of fetal, neonatal, and post-neonatal death compared to live infants (reference group).

Table 4 presents the adjusted analysis of the risk of fetal, neonatal, and post-neonatal deaths compared to live infants (reference group). The final analysis model showed a strong positive association between fetal, neonatal, and post-neonatal death [odds ratio (OR): 15.60, 7.63, and 5.51, respectively] and less than six prenatal care consultations. Also, fetal deaths were more likely to occur with nonwhite mothers compared to live infants. Neonatal deaths were 14 times more frequent in infants with preterm delivery (37 weeks or less) compared to live infants. When compared to live infants, infants who were born in a C-section delivery and who was offspring of mothers with low schooling had increased odds of post-neonatal death. Neonatal deaths were 102.4 times more likely to have a low 5th minute Apgar score. 


\section{Table 1}

Fetal, neonatal, and post-neonatal and infant mortality in the 2015 Pelotas (Brazil) birth cohort.

\begin{tabular}{|c|c|}
\hline Total births $(N=4,329)$ & \\
\hline \multicolumn{2}{|l|}{ Fetal deaths* } \\
\hline Number & 54 \\
\hline Fetal mortality rate 1,000 births $(95 \% \mathrm{Cl})$ & $12.5(9.2-15.8)$ \\
\hline \multicolumn{2}{|l|}{ Neonatal deaths } \\
\hline Number & 37 \\
\hline Neonatal mortality rate/1,000 live births $(95 \% \mathrm{Cl})$ & $8.7(5.8-11.4)$ \\
\hline \multicolumn{2}{|l|}{ Post-neonatal deaths } \\
\hline Number & 22 \\
\hline Post-neonatal mortality rate/1,000 live births $(95 \% \mathrm{Cl})$ & $5.1(3.0-7.3)$ \\
\hline \multicolumn{2}{|l|}{ Infant's deaths } \\
\hline Number & 59 \\
\hline Infant mortality rate/1,000 live births $(95 \% \mathrm{Cl})$ & $13.8(10.3-17.3)$ \\
\hline
\end{tabular}

Table 2

Sociodemographic and health characteristics of mothers and children in the general sample and deaths of the 2015 Pelotas (Brazil) birth cohort.

\begin{tabular}{|c|c|c|c|c|c|c|c|c|c|c|c|}
\hline \multirow[t]{2}{*}{ Characteristics } & \multirow{2}{*}{$\begin{array}{c}\text { Total } \\
\text { sample } \\
(4,275 \text { live } \\
\text { births) } \\
\text { n (\%) }\end{array}$} & \multicolumn{2}{|c|}{$\begin{array}{l}\text { Fetal deaths } \\
\quad(n=54)\end{array}$} & \multicolumn{2}{|c|}{$\begin{array}{l}\text { Neonatal deaths } \\
\quad(n=37)\end{array}$} & \multicolumn{2}{|c|}{$\begin{array}{l}\text { Post-neonatal } \\
\text { deaths }(n=22)\end{array}$} & \multicolumn{2}{|c|}{$\begin{array}{l}\text { Infant deaths } \\
\quad(n=59)\end{array}$} & \multicolumn{2}{|c|}{$\begin{array}{l}\text { Total deaths } \\
\quad(n=113)\end{array}$} \\
\hline & & n (\%) & p-value & n (\%) & p-value & n (\%) & p-value & n (\%) & p-value & n (\%) & p-value \\
\hline $\begin{array}{l}\text { Distal level: } \\
\text { maternal } \\
\text { characteristics } \\
\text { during pregnancy }\end{array}$ & & & & & & & & & & & \\
\hline \multicolumn{12}{|l|}{ Age (years) } \\
\hline$\geq 20$ & $3,652(85.5)$ & $44(81.5)$ & & $32(86.5)$ & & $15(68.2)$ & & 47 (79.7) & & $91(80.5)$ & \\
\hline \multicolumn{12}{|l|}{ Skin color } \\
\hline White & $3,071(71.9)$ & $28(51.9)$ & 0.001 & $23(62.2)$ & 0.185 & $13(59.1)$ & 0.179 & $23(39.0)$ & 0.062 & 64 (56.6) & $<0.001$ \\
\hline Non-white & $1,203(28.2)$ & $26(48.1)$ & - & $14(37.8)$ & - & $9(40.9)$ & - & $36(61.0)$ & - & $49(43.4)$ & - \\
\hline \multicolumn{12}{|l|}{ Wealth index } \\
\hline Category A/B & $1,275(30.8)$ & $41(82.0)$ & 0.045 & $3(8.3)$ & 0.003 & $1(5.0)$ & 0.012 & $4(7.1)$ & $<0.001$ & $13(12.3)$ & $<0.001$ \\
\hline Category C/D/E & $2,855(69.1)$ & $9(18.0)$ & - & $33(91.7)$ & - & $19(95.0)$ & - & $52(96.9)$ & - & $93(87.7)$ & - \\
\hline \multicolumn{12}{|l|}{$\begin{array}{l}\text { Maternal education } \\
\text { (complete years of } \\
\text { schooling) }\end{array}$} \\
\hline$\leq 5$ & 655 (15.3) & $13(24.1)$ & 0.066 & $27(73.0)$ & 0.043 & $11(50.0)$ & $<0.001$ & 21 (35.6) & $<0.001$ & $34(30.1)$ & $<0.001$ \\
\hline$>6$ & $3,619(84.7)$ & 41 (75.9) & - & $10(27.0)$ & - & $11(50.0)$ & - & $38(64.4)$ & - & 79 (69.9) & - \\
\hline
\end{tabular}

(continues) 
Table 2 (continued)

\begin{tabular}{|c|c|c|c|c|c|c|c|c|c|c|c|}
\hline \multirow[t]{2}{*}{ Characteristics } & \multirow{2}{*}{$\begin{array}{c}\text { Total } \\
\text { sample } \\
(4,275 \text { live } \\
\text { births) } \\
\text { n (\%) }\end{array}$} & \multicolumn{2}{|c|}{$\begin{array}{l}\text { Fetal deaths } \\
\quad(n=54)\end{array}$} & \multicolumn{2}{|c|}{$\begin{array}{l}\text { Neonatal deaths } \\
\quad(n=37)\end{array}$} & \multicolumn{2}{|c|}{$\begin{array}{l}\text { Post-neonatal } \\
\text { deaths }(n=22)\end{array}$} & \multicolumn{2}{|c|}{$\begin{array}{l}\text { Infant deaths } \\
\quad(n=59)\end{array}$} & \multicolumn{2}{|c|}{$\begin{array}{l}\text { Total deaths } \\
\qquad(n=113)\end{array}$} \\
\hline & & n (\%) & p-value & n (\%) & p-value & n (\%) & p-value & n (\%) & p-value & n (\%) & p-value \\
\hline \multicolumn{12}{|l|}{ Intermediate } \\
\hline \multicolumn{12}{|l|}{ level I: maternal } \\
\hline \multicolumn{12}{|l|}{ characteristics } \\
\hline \multicolumn{12}{|l|}{ during perinatal } \\
\hline \multicolumn{12}{|l|}{ period } \\
\hline \multicolumn{12}{|l|}{ Marital status } \\
\hline Without partner & $607(14.2)$ & $20(37.0)$ & $<0.001$ & $7(18.9)$ & 0.399 & $7(31.8)$ & 0.017 & $14(23.7)$ & 0.035 & $34(30.1)$ & $<0.001$ \\
\hline With partner & $3,667(85.8)$ & $34(63.0)$ & - & $30(81.1)$ & - & $15(68.2)$ & - & $45(76.3)$ & - & 79 (69.9) & - \\
\hline \multicolumn{12}{|l|}{ Infectious diseases } \\
\hline No & $2,283(53.4)$ & $30(55.6)$ & 0.757 & $16(43.2)$ & 0.216 & $14(63.6)$ & 0.339 & $30(50.9)$ & 0.692 & $60(53.1)$ & 0.943 \\
\hline Yes & $1,992(46.6)$ & $24(44.4)$ & - & $21(56.8)$ & - & $8(36.4)$ & - & $29(49.1)$ & - & $53(46.9)$ & - \\
\hline \multicolumn{12}{|l|}{$\begin{array}{l}\text { Cardiovascular } \\
\text { diseases }\end{array}$} \\
\hline No & $3,624(84.8)$ & $49(90.7)$ & 0.238 & $21(56.8)$ & $<0.001$ & $20(90.9)$ & 0.438 & $41(69.5)$ & 0.001 & 90 (79.7) & 0.188 \\
\hline Yes & $651(15.2)$ & $5(9.3)$ & - & $16(43.2)$ & - & $2(9.1)$ & - & $18(30.5)$ & - & $23(20.4)$ & - \\
\hline \multicolumn{12}{|l|}{ Anaemia ** } \\
\hline No & $2,461(57.7)$ & $34(63.0)$ & 0.414 & 33 (91.7) & $<0.001$ & $10(45.5)$ & 0.257 & $43(74.1)$ & 0.011 & $77(68.8)$ & 0.017 \\
\hline Yes & $1,807(42.3)$ & $20(37.0)$ & - & $3(8.3)$ & - & $12(54.6)$ & - & $15(25.9)$ & - & $35(31.3)$ & - \\
\hline \multicolumn{12}{|l|}{$\begin{array}{l}\text { Abortion threat or } \\
\text { premature delivery }\end{array}$} \\
\hline No & $3,074(71.9)$ & $36(66.7)$ & 0.363 & $14(37.8)$ & $<0.001$ & $14(63.6)$ & 0.368 & $28(47.5)$ & $<0.001$ & $64(56.6)$ & $<0.001$ \\
\hline Yes & $1,201(28.1)$ & $18(33.3)$ & - & $23(62.2)$ & - & $8(36.4)$ & - & $31(52.5)$ & - & $49(43.4)$ & - \\
\hline \multicolumn{12}{|c|}{$\begin{array}{l}\text { Depression or mental } \\
\text { health problems }\end{array}$} \\
\hline No & $3,771(88.3)$ & $40(74.1)$ & $<0.001$ & $31(83.8)$ & 0.392 & $18(81.8)$ & 0.344 & $49(83.1)$ & 0.212 & $89(78.8)$ & 0.002 \\
\hline Yes & $502(11.8)$ & $14(25.9)$ & - & $6(16.2)$ & & $4(18.2)$ & & $10(16.9)$ & & $24(21.24)$ & \\
\hline \multicolumn{12}{|l|}{$\begin{array}{l}\text { Smoking or alcohol } \\
\text { history }\end{array}$} \\
\hline No & $3,371(78.9)$ & $34(63.0)$ & 0.004 & $25(67.6)$ & 0.087 & $12(54.6)$ & 0.005 & $37(62.7)$ & 0.002 & $71(62.8)$ & $<0.001$ \\
\hline Yes & $904(21.2)$ & $20(37.0)$ & - & $12(32.4)$ & - & $10(45.5)$ & - & $22(37.3)$ & - & $42(37.7)$ & - \\
\hline \multicolumn{12}{|c|}{$\begin{array}{l}\text { Adequate weight gain } \\
\text { during pregnancy }\end{array}$} \\
\hline No & $2,777(65.0)$ & $10(18.5)$ & 0.011 & $10(27.0)$ & 0.304 & $7(31.8)$ & 0.746 & $42(71.2)$ & 0.313 & $86(76.1)$ & 0.013 \\
\hline Yes & $1,498(35.0)$ & $44(81.5)$ & - & $27(73.0)$ & - & $15(68.2)$ & - & $17(28.8)$ & - & 27 (23.9) & - \\
\hline \multicolumn{12}{|l|}{ Parity } \\
\hline Primiparous & $2,136(50.0)$ & $35(64.8)$ & 0.032 & $14(37.8)$ & 0.138 & $11(50.0)$ & 0.993 & $25(42.4)$ & 0.239 & $60(53.1)$ & 0.529 \\
\hline$\geq 2$ children & $2,137(50.0)$ & $19(35.2)$ & - & $23(62.2)$ & - & $11(50.0)$ & - & $34(57.6)$ & - & 53 (46.9) & - \\
\hline \multicolumn{12}{|l|}{ Abortion history } \\
\hline No & $1,192(68.2)$ & $4(30.8)$ & 0.004 & $7(58.3)$ & 0.463 & $6(85.7)$ & 0.321 & $13(68.4)$ & 0.986 & $17(53.1)$ & 0.070 \\
\hline Yes & $555(31.8)$ & $9(69.2)$ & - & $5(41.7)$ & - & $1(14.3)$ & - & $6(31.6)$ & - & $15(46.9)$ & - \\
\hline \multicolumn{12}{|l|}{ Twin pregnancy } \\
\hline No & 2,715 (96.9) & $20(100.0)$ & 0.432 & $14(82.3)$ & $<0.001$ & $11(91.7)$ & 0.280 & $25(86.2)$ & 0.001 & $45(91.8)$ & 0.038 \\
\hline Yes & $87(3.1)$ & $0(0.0)$ & - & $3(17.7)$ & - & $1(8.3)$ & - & $4(13.8)$ & - & $4(8.2)$ & - \\
\hline
\end{tabular}

(continues) 
Table 2 (continued)

\begin{tabular}{|c|c|c|c|c|c|c|c|c|c|c|c|}
\hline \multirow[t]{2}{*}{ Characteristics } & \multirow{2}{*}{$\begin{array}{c}\text { Total } \\
\text { sample } \\
(4,275 \text { live } \\
\text { births) } \\
n(\%)\end{array}$} & \multicolumn{2}{|c|}{$\begin{array}{l}\text { Fetal deaths } \\
\qquad(\mathrm{n}=54)\end{array}$} & \multicolumn{2}{|c|}{$\begin{array}{l}\text { Neonatal deaths } \\
\qquad(\mathrm{n}=37)\end{array}$} & \multicolumn{2}{|c|}{$\begin{array}{l}\text { Post-neonatal } \\
\text { deaths }(n=22)\end{array}$} & \multicolumn{2}{|c|}{$\begin{array}{l}\text { Infant deaths } \\
\qquad(n=59)\end{array}$} & \multicolumn{2}{|c|}{$\begin{array}{l}\text { Total deaths } \\
\qquad(n=113)\end{array}$} \\
\hline & & n (\%) & p-value & n (\%) & p-value & n (\%) & p-value & n (\%) & p-value & n (\%) & p-value \\
\hline \multicolumn{12}{|l|}{ Intermediate } \\
\hline \multicolumn{12}{|l|}{ level II: maternal } \\
\hline \multicolumn{12}{|l|}{ characteristics } \\
\hline \multicolumn{12}{|l|}{ during perinatal } \\
\hline \multicolumn{12}{|l|}{ period } \\
\hline \multirow{2}{*}{\multicolumn{12}{|c|}{$\begin{array}{l}\text { Number of prenatal } \\
\text { care consultations }\end{array}$}} \\
\hline & & & & & & & & & & & \\
\hline$<6$ & $586(14.1)$ & $28(65.1)$ & $<0.001$ & $23(69.7)$ & $<0.001$ & $9(45.0)$ & $<0.001$ & $34(64.2)$ & $<0.001$ & $62(64.6)$ & $<0.001$ \\
\hline$\geq 6$ & $3,583(85.9)$ & 15 (34.9) & - & $10(30.3)$ & - & $11(55.0)$ & - & 19 (35.9) & - & 34 (35.4) & - \\
\hline \multicolumn{12}{|l|}{ Type of delivery } \\
\hline Normal & $1,489(34.8)$ & - & - & $16(43.2)$ & 0.286 & $4(18.2)$ & 0.102 & 20 (33.9) & 0.879 & $51(45.1)$ & 0.024 \\
\hline C-section & $2,785(65.2)$ & - & - & $21(56.8)$ & - & $18(81.8)$ & - & $39(66.1)$ & - & 62 (54.9) & - \\
\hline \multicolumn{12}{|c|}{$\begin{array}{l}\text { Professional who } \\
\text { attended the delivery }\end{array}$} \\
\hline Physician & $4,096(96.5)$ & - & - & $33(89.2)$ & 0.016 & $22(100.0)$ & 0.374 & $55(93.2)$ & 0.169 & 105 (92.9) & $<0.001$ \\
\hline \multicolumn{12}{|l|}{$\begin{array}{l}\text { Same professional } \\
\text { performed delivery } \\
\text { and prenatal } \\
\text { consultations }\end{array}$} \\
\hline No & $1,956(46.7)$ & - & - & $19(57.6)$ & 0.62 & $10(50.0)$ & 0.771 & $24(45.3)$ & 0.832 & $48(50.0)$ & 0.528 \\
\hline Yes & $2,230(53.3)$ & - & & $14(42.4)$ & & $10(50.0)$ & & $29(54.7)$ & & $48(50.0)$ ** & \\
\hline \multicolumn{12}{|c|}{$\begin{array}{l}\text { Proximal level: child } \\
\text { characteristics }\end{array}$} \\
\hline \multicolumn{12}{|l|}{ Sex } \\
\hline Male & 2,165 (50.6) & - & - & $21(56.8)$ & 0.46 & $8(36.4)$ & 0.181 & $29(49.2)$ & 0.818 & $61(54.0)$ & 0.486 \\
\hline Female & $2,110(49.4)$ & - & - & $16(43.2)$ & - & $14(63.6)$ & - & 30 (50.9) & - & $52(46.0)$ & - \\
\hline \multicolumn{12}{|l|}{ Gestational age ** } \\
\hline Term & $3,393(82.0)$ & - & - & $26(86.7)$ & $<0.001$ & $10(52.6)$ & $<0.001$ & 35 (71.4) & $<0.001$ & $70(76.9) * *$ & $<0.001$ \\
\hline Preterm & $747(18.0)$ & - & & $4(13.3)$ & - & $9(47.4)$ & - & $14(28.6)$ & - & $21(23.08)$ & - \\
\hline \multicolumn{12}{|l|}{ Birth weight (g) } \\
\hline$\leq 2,500$ & $428(10.0)$ & - & - & $20(54.1)$ & $<0.001$ & $8(36.4)$ & $<0.001$ & $28(47.5)$ & $<0.001$ & $28(47.5)$ & $<0.001$ \\
\hline$>2,500$ & $3,844(90.0)$ & - & - & $17(46.0)$ & - & $14(63.6)$ & - & $31(52.5)$ & - & $31(52.5)$ & - \\
\hline \multicolumn{12}{|l|}{$\begin{array}{l}\text { Apgar } 5 \text { th } \\
\text { minute } * *, * * *\end{array}$} \\
\hline$<7$ & $69(1.6)$ & - & - & $16(47.1)$ & $<0.001$ & $1(4.6)$ & 0.069 & $17(30.1)$ & $<0.001$ & - & - \\
\hline 7-10 (adequate) & $\begin{array}{c}4,206(98.4) \\
(91.3)\end{array}$ & - & - & $18(52.9)$ & - & $21(95.5)$ & - & $39(69.6)$ & - & - & - \\
\hline
\end{tabular}

* Brazilian wealth index classification (Associação Brasileira de Empresas de Pesquisa. http://www.abep.org);

** The missing values of the variable cause the total value not to be 113 ;

$* * *$ Comparison with the general sample as a reference group $(n=4,275)$. 
Table 3

Multinomial logistic regression showing the crude risk of fetal, neonatal, and post-neonatal death compared to the general sample of the 2015 Pelotas (Brazil) birth cohort.

\begin{tabular}{|c|c|c|c|c|c|c|c|}
\hline \multirow[t]{2}{*}{ Characteristics } & \multicolumn{2}{|c|}{$\begin{array}{l}\text { Fetal deaths } \\
\qquad(n=54) \text { * }\end{array}$} & \multicolumn{2}{|c|}{$\begin{array}{c}\text { Neonatal deaths } \\
(\mathrm{n}=37) \text { * }\end{array}$} & \multicolumn{2}{|c|}{$\begin{array}{l}\text { Post-neonatal deaths } \\
\qquad(n=22) \text { * }\end{array}$} & \multirow[t]{2}{*}{ p-value } \\
\hline & $\begin{array}{l}\text { Unadjusted } \\
\text { OR }\end{array}$ & $95 \% \mathrm{Cl}$ & $\begin{array}{l}\text { Unadjusted } \\
\text { OR }\end{array}$ & $95 \% \mathrm{Cl}$ & $\begin{array}{l}\text { Unadjusted } \\
\text { OR }\end{array}$ & $95 \% \mathrm{Cl}$ & \\
\hline \multicolumn{8}{|l|}{$\begin{array}{l}\text { Distal level: maternal characteristics } \\
\text { during pregnancy }\end{array}$} \\
\hline Age (years) & & & & & & & 0.182 \\
\hline$\geq 20$ & 1.00 & - & 1.00 & - & 1.00 & - & \\
\hline Skin color & & & & & & & 0.005 \\
\hline White & 1.00 & - & 1.00 & - & 1.00 & - & \\
\hline Non-white & 2.39 & $1.39-4.09$ & 1.56 & $0.80-3.05$ & 1.78 & $0.76-4.18$ & \\
\hline Wealth index (ABEP) ** & & & & & & & $<0.001$ \\
\hline Category A/B & 1.00 & - & 1.00 & - & 1.00 & - & \\
\hline Category C/D/E & 2.06 & $1.00-4.26$ & 4.99 & $1.53-16.29$ & 8.61 & $1.15-64.42$ & \\
\hline $\begin{array}{l}\text { Maternal education (complete years of } \\
\text { schooling) }\end{array}$ & & & & & & & $<0.001$ \\
\hline$\leq 5$ & 1.79 & $0.95-3.36$ & 2.09 & $1.01-4.34$ & 5.65 & $2.44-13.08$ & \\
\hline$>6$ & 1.00 & - & 1.00 & - & 1.00 & - & \\
\hline \multicolumn{8}{|l|}{$\begin{array}{l}\text { Intermediate level I: maternal } \\
\text { characteristics during perinatal period }\end{array}$} \\
\hline Marital status & & & & & & & $<0.001$ \\
\hline Without partner & 3.59 & $2.05-6.28$ & 1.43 & $0.62-3.26$ & 2.85 & $1.16-7.02$ & \\
\hline With partner & 1.00 & - & 1.00 & - & 1.00 & - & \\
\hline Infectious diseases & & & & & & & 0.462 \\
\hline No & 1.00 & - & 1.00 & - & 1.00 & - & \\
\hline Yes & 0.92 & $0.53-1.57$ & 1.50 & $0.78-2.89$ & 0.66 & $0.27-1.57$ & \\
\hline Cardiovascular diseases & & & & & & & $<0.001$ \\
\hline No & 1.00 & - & 1.00 & - & 1.00 & - & \\
\hline Yes & 0.58 & $0.23-1.46$ & 4.31 & $2.24-8.31$ & 0.57 & $0.13-2.43$ & \\
\hline Anaemia & & & & & & & $<0.001$ \\
\hline No & 1.00 & - & 1.00 & - & 1.00 & - & \\
\hline Yes & 0.79 & $0.46-1.38$ & 0.12 & $0.04-0.40$ & 1.62 & $0.70-3.76$ & \\
\hline Abortion threat or premature delivery & & & & & & & $<0.001$ \\
\hline No & 1.00 & - & 1.00 & - & 1.00 & - & \\
\hline Yes & 1.30 & $0.74-2.30$ & 4.28 & $2.19-8.34$ & 1.49 & $0.62-3.56$ & \\
\hline Depression or mental health problems & & & & & & & 0.024 \\
\hline No & 1.00 & - & 1.00 & - & 1.00 & - & \\
\hline Yes & 2.65 & $1.43-4.90$ & 1.46 & $0.61-3.53$ & 1.68 & $0.57-4.99$ & \\
\hline Smoking or alcohol history & & & & & & & 0.011 \\
\hline No & 1.00 & - & 1.00 & - & 1.00 & - & \\
\hline Yes & 2.22 & $1.27-3.88$ & 1.81 & $0.91-3.63$ & 3.15 & $1.36-7.31$ & \\
\hline Adequate weight gain during pregnancy & & & & & & & 0.040 \\
\hline No & 2.38 & $1.19-4.75$ & 1.46 & $0.71-3.03$ & 1.16 & $0.47-2.85$ & \\
\hline Yes & 1.00 & - & 1.00 & - & 1.00 & - & \\
\hline Parity & & & & & & & 0.070 \\
\hline Primiparous & 1.00 & - & 1.00 & - & 1.00 & - & \\
\hline$\geq 2$ children & 0.54 & $0.31-0.96$ & 1.65 & $0.85-3.21$ & 1.00 & $0.43-2.32$ & \\
\hline
\end{tabular}


Table 3 (continued)

\begin{tabular}{|c|c|c|c|c|c|c|c|}
\hline \multirow[t]{2}{*}{ Characteristics } & \multicolumn{2}{|c|}{$\begin{array}{l}\text { Fetal deaths } \\
(n=54) *\end{array}$} & \multicolumn{2}{|c|}{$\begin{array}{l}\text { Neonatal deaths } \\
\quad(n=37) *\end{array}$} & \multicolumn{2}{|c|}{$\begin{array}{l}\text { Post-neonatal deaths } \\
\qquad(n=22) *\end{array}$} & \multirow[t]{2}{*}{ p-value } \\
\hline & $\begin{array}{l}\text { Unadjusted } \\
\text { OR }\end{array}$ & $95 \% \mathrm{Cl}$ & $\begin{array}{l}\text { Unadjusted } \\
\text { OR }\end{array}$ & $95 \% \mathrm{Cl}$ & $\begin{array}{l}\text { Unadjusted } \\
\text { OR }\end{array}$ & $95 \% \mathrm{Cl}$ & \\
\hline \multicolumn{8}{|l|}{ Intermediate level I: maternal } \\
\hline Abortion history & & & & & & & 0.030 \\
\hline No & 1.00 & - & 1.00 & - & 1.00 & - & \\
\hline Yes & 4.83 & $1.48-15.76$ & 1.53 & $0.48-4.85$ & 0.36 & $0.043-2.98$ & \\
\hline Twin pregnancy & & & & & & & 0.046 \\
\hline No & - & - & 1.00 & - & 1.00 & - & \\
\hline Yes & - & - & 6.94 & $1.96-24.63$ & 2.95 & $0.38-23.09$ & \\
\hline \multicolumn{8}{|l|}{$\begin{array}{l}\text { Intermediate level II: maternal } \\
\text { characteristics during perinatal period }\end{array}$} \\
\hline Number of prenatal care consultations & & & & & & & $<0.001$ \\
\hline$<6$ & 12.05 & $6.40-22.71$ & 14.85 & $7.03-31.36$ & 7.89 & $3.26-19.12$ & \\
\hline$\geq 6$ & 1.00 & - & 1.00 & - & 1.00 & - & \\
\hline Type of delivery & & & & & & & 0.002 \\
\hline Normal & - & - & 1.42 & $0.74-2.74$ & 4.15 & $0.14-1.23$ & \\
\hline C-section & - & & 1.00 & - & 1.00 & - & \\
\hline Professional who attended the delivery & & & & & & & 0.111 \\
\hline Physician & - & - & 1.00 & - & - & - & \\
\hline $\begin{array}{l}\text { Non-physician (nurse, student, } \\
\text { midwife, other) }\end{array}$ & - & - & 3.38 & $1.18-9.66$ & - & - & \\
\hline \multicolumn{8}{|l|}{ and prenatal consultations } \\
\hline No & - & - & 0.84 & $0.42-1.68$ & 1.14 & $0.47-2.74$ & \\
\hline Yes & - & - & 1.00 & - & 1.00 & - & \\
\hline \multicolumn{8}{|l|}{ Proximal level: child characteristics } \\
\hline Sex & & & & & & & 0.265 \\
\hline Male & 1.00 & - & 1.00 & - & 1.00 & - & \\
\hline Female & 0.71 & $0.41-1.21$ & 0.78 & $0.41-1.50$ & 1.80 & $0.75-4.29$ & \\
\hline Gestational age & & & & & & & $<0.001$ \\
\hline Term & - & - & 1.00 & - & 1.00 & - & \\
\hline Preterm & - & - & 30.85 & $10.73-88.67$ & 4.27 & $1.73-10.55$ & \\
\hline \multicolumn{8}{|l|}{ Birth weight (g) } \\
\hline$\leq 2,500$ & - & - & 11.21 & $5.83-21.58$ & 5.45 & $2.27-13.06$ & $<0.001$ \\
\hline$>2,500$ & - & & 1.00 & - & 1.00 & - & \\
\hline \multicolumn{8}{|l|}{ Apgar 5th minute } \\
\hline$<7$ & - & - & 100.1 & $47.43-211.35$ & 5.36 & $0.70-40.91$ & $<0.001$ \\
\hline 7-10 (adequate) & - & & 1.00 & - & 1.00 & - & \\
\hline
\end{tabular}

95\%Cl: 95\% confidence interval; OR: odds ratio.

* Comparison with the general sample as a reference group $(n=4,275)$;

** Brazilian wealth index classification (Associação Brasileira de Empresas de Pesquisa. http://www.abep.org). 


\section{Table 4}

Multinomial logistic regression presenting the adjusted risk of fetal, neonatal, and post-neonatal mortality compared to the general sample of the 2015 Pelotas (Brazil) birth cohort.

\begin{tabular}{|c|c|c|c|c|c|c|}
\hline \multirow[t]{2}{*}{ Characteristics } & \multicolumn{2}{|c|}{ Fetal deaths $(n=54)$ * } & \multicolumn{2}{|c|}{ Neonatal deaths $(n=37)$ * } & \multicolumn{2}{|c|}{ Post-neonatal deaths $(n=22)$ * } \\
\hline & Adjusted OR & $95 \% \mathrm{Cl}$ & Adjusted OR & $95 \% \mathrm{Cl}$ & Adjusted OR & $95 \% \mathrm{Cl}$ \\
\hline \multicolumn{7}{|c|}{ Distal level: maternal characteristics } \\
\hline \multicolumn{7}{|c|}{ during pregnancy } \\
\hline \multicolumn{7}{|l|}{ Skin color } \\
\hline White & 1.00 & - & 1.00 & - & 1.00 & - \\
\hline No white & 8.25 & $1.21-56.03$ & 1.58 & $0.62-4.03$ & 0.68 & $0.23-2.07$ \\
\hline \multicolumn{7}{|c|}{$\begin{array}{l}\text { Maternal education (complete years of } \\
\text { schooling) }\end{array}$} \\
\hline$\leq 5$ & 0.08 & $0.01-1.15$ & 1.03 & $0.34-3.10$ & 3.86 & $1.33-11.23$ \\
\hline$>6$ & 1.00 & - & 1.00 & - & 1.00 & - \\
\hline \multicolumn{7}{|c|}{ Intermediate level I: maternal } \\
\hline \multicolumn{7}{|c|}{ characteristics during perinatal period } \\
\hline \multicolumn{7}{|c|}{ Number of antenatal care consultations } \\
\hline$<6$ & 15.60 & $1.71-142.13$ & 7.63 & 2.91-19.99 & 5.51 & $1.95-15.56$ \\
\hline$\geq 6$ & 1.00 & - & 1.00 & - & 1.00 & - \\
\hline \multicolumn{7}{|c|}{ Intermediate level II: maternal } \\
\hline \multicolumn{7}{|c|}{ characteristics during perinatal period } \\
\hline \multicolumn{7}{|c|}{ Type of delivery } \\
\hline Normal & - & - & 1.00 & - & 1.00 & - \\
\hline C-section & - & - & 2.18 & $0.80-5.95$ & 3.71 & $1.02-13.43$ \\
\hline \multicolumn{7}{|c|}{ Proximal level: child characteristics } \\
\hline \multicolumn{7}{|l|}{ Gestational age ** } \\
\hline Term & - & - & 1.00 & - & - & - \\
\hline Preterm & - & - & 14.09 & $4.51-44.07$ & 3.54 & $1.29-9.68$ \\
\hline \multicolumn{7}{|l|}{ Apgar 5 th minute } \\
\hline$<7$ & - & - & 102.37 & $34.18-306.61$ & 7.58 & $0.86-67.22$ \\
\hline 7-10 (adequate) & - & - & 1.00 & - & 1.00 & - \\
\hline
\end{tabular}

* Comparison with the general sample as a reference group $(n=4,275)$;

** Estimated by the cohort.

\section{Discussion}

In this study, an FMR of 12.5 stillbirths/1,000 births and an IMR of 13.8 deaths/1,000 live births was estimated. Insufficient prenatal visits (six or less) was the factor associated with an increased risk of fetal, neonatal, and post-neonatal death. Preterm birth and a low Apgar score in the fifth-minute of life after birth were also associated with neonatal and post-neonatal deaths. Also, the lower maternal education increased about four times the risk of post-neonatal deaths in Pelotas.

The city of Pelotas, State of Rio Grande do Sul, in the South region of Brazil, has a population of about 340,000 inhabitants and has a series of cohort studies (1982, 1993, 2004, and 2015). The cohort data show that antepartum fetal deaths decreased from 13.1/1,000 births in 1982 to 8.4/1,000 in 2004. The IMR was 36.4/1,000 births in 1982 and 19.4/1,000 in 2004.

FMR is considered one of the best indicators of the quality of care provided to pregnant women and during childbirth 22, but is not included by the United Nations as one of the Millennium Development Goals 23, therefore, fetal deaths are not included in health policies. Developed countries present a low FMR, ranging from two to seven per thousand births, in contrast to Brazil, where the minimum FMR minimum was 7.7/1,000 in the South and 12.1/1,000 in the Northeast. Fetal deaths have decreased in Brazil; however, inequalities persist. The causes of death appointed by the literature 
indicate maternal morbidities that could be prevented and treated. The main factors associated with fetal deaths were absent or inadequate prenatal care, low education level, maternal morbidity, and adverse reproductive history ${ }^{24}$. Froen et al. 25 showed that the absence of prenatal care was the main risk factor for fetal death in seven low or middle-income countries.

The IMR in Brazil is 12.35/1,000 births in 2018 (Instituto Brasileiro de Geografia e Estatística. Cidades e estados. Mortalidade infantil. https://www.ibge.gov.br/informacoes-por-cidade-e-estado. html? $\mathrm{t}=$ destaques\&c=Brasil, accessed on 12/Nov/2018). The World Health Organization (WHO) considers the IMR to analyze access, use, and effectiveness of health care, particularly prenatal, delivery, newborn, and child care in the first year of life 26 . The findings of this study are consistent with other studies in Brazil 27,28. Recent evidence indicates that a higher frequency of prenatal consultations with a health professional is associated with a lower probability of maternal and infant death, which could be due to increased opportunities to detect and manage potential complications. Also, the literature has reported that prenatal care visits decrease the risk of neonatal mortality [pooled effect size 0.66 (95\%CI: 0.54-0.80)] and that the risk of neonatal death was significantly reduced by $34 \%$ among newborns delivered from mothers who had prenatal care visits 29 .

According to the WHO in 2014, six or more prenatal care contacts vs. four visits can reduce perinatal deaths by up to 8/1,000 births 26,30. In 2015, the Brazilian Ministry of Health recommended a minimum number of six visits for a full-term pregnancy, with prenatal care starting in the first trimester with basic procedures and including clinical-obstetrical and laboratory tests 31 . In Brazil, prenatal care adequacy is around 45\% when assessed in terms of onset and number of consultations 22 . Current programs such as the Prenatal and Birth Humanization Program (PHPN) represent a strategy to implement qualitative interventions to improve prenatal adequacy, such as including guidelines on breastfeeding, supplementary feeding, and immunization, among others in the country 26,31 .

Infant mortality studies show that deaths during the neonatal period are more likely associated with genetic, pregnancy, type of delivery, and perinatal characteristics, while post-neonatal mortality is related to factors determined by living conditions and social characteristics, such as income, education, basic sanitation and treated clean water, and access to health services $8,12,14$. Neonatal mortality in Brazil accounts for more than $70 \%$ of mortality in the first year of life, with $25 \%$ of deaths occurring in the first 24 hours postpartum 17,18,19. Based on these data, it was verified in this study that $32.7 \%$ of the deaths were neonates and that those born prematurely had an increased risk of death in this period compared to those born at term.

Studies have shown that women who did not attend or who had a low number of prenatal care consultations had a higher risk of preterm birth, an outcome that is related to high infant mortality 32,33. Kilsztajn et al. 34 showed that the increase in the number of prenatal consultations from 0 to 3 to 7 or more significantly reduced the prevalence of preterm delivery (from 14\% to 4\%). In addition, women who received an insufficient number of visits (OR $=1.99 ; 95 \% \mathrm{CI}$ : 1.20-3.28) and started prenatal care after the $16^{\text {th }}$ week of gestation $(\mathrm{OR}=1.94$; 95\%CI: 1.12-3.34) had almost double the likelihood of a preterm birth compared to those who started prenatal care early and attended an adequate number of visits (at least six visits) 34 .

A low Apgar score in the $5^{\text {th }}$ minute of life was another characteristic associated with neonatal and post-neonatal death in this study. The low vitality of newborns is a factor that has been pointed out in studies as a predictor of neonatal mortality, emphasizing the importance of an adequate labor surveillance and quality of care provided to the newborn as a way to reduce death in the perinatal period 35. An international study showed a low (0-3) 5th minute Apgar score was associated with an adjusted relative risk of 359.4 (95\%CI: 277.3-465.9) for early neonatal death and 30.5 (95\%CI: 18.0 51.6) for late neonatal death 36 .

A cohort study conducted in Santa Catarina, Brazil, identified an IMR of 7.7/1,000 live births. Also, a pregnancy duration of less than 32 weeks was among the risk factors associated with increased risk of death in the first year of life $(\mathrm{OR}=12.08) 28$.

In this study, participants with non-white skin color and schooling less or equal to five years had eight- and four-times higher risk of fetal and post-neonatal deaths, respectively. In Brazil, despite increasing coverage of prenatal care since the 1990s 37,38, with the requirement of at least six consultations and effective procedures recommended by the Brazilian Ministry of Health 22 , it is important to 
consider the application of these procedures to reduce adverse pregnancy and birth outcomes among mothers and newborns 39,40 .

This study has the strength of being a cohort study including primary collection of mortality data in the first year of life with simultaneous monitoring of several sources of mortality and morbidity information. Limitations include the possibility of error in the measurement of exposures due to the maternal self-reporting of specific variables.

\section{Conclusion}

This study showed that an insufficient number of prenatal care consultations increased the odds of fetal and infant mortality. Specifically, non-white skin color, low maternal education, preterm birth, C-section delivery, and low Apgar score in the $5^{\text {th }}$ minute of life increased the odds of infant deaths. These findings show the need for early intervention during pregnancy, thus ensuring access to adequate prenatal care and contributing to the prevention of infant mortality.

\section{Contributors}

A. R. Varela, B. C. Schneider, S. Bubach, M. F. Silveira, A. D. Bertoldi and D. G. Bassani were responsible for proposing the initial hypothesis and idea for study, designing the study, writing the first draft of the manuscript and analyzing and interpreting the data; contributed to the critical review of the final version of this manuscript, provided feedback on drafts, and approved the final version. L. S. M. Duarte contributed to the critical review of the final version of this manuscript, provided feedback on drafts, and approved the final version. A. M. B. Menezes contributed to the interpretation and consistency of the data and the critical review of the final version of this manuscript, provided feedback on drafts, and approved the final version. M. R. Domingues was responsible for planning the cohort in which this sub-study was carried out; contributed to the critical review of the final version of this manuscript, provided feedback on drafts, and approved the final version.

\section{Additional informations}

ORCID: Andrea Ramirez Varela (0000-00032685-9617); Bruna Celestino Schneider (00000002-1035-0106); Susana Bubach (0000-00027190-5275); Mariangela Freitas Silveira (00000002-2861-7139); Andréa Dâmaso Bertoldi (0000-0002-4680-3197); Lina Sofia Morón-Duarte (0000-0002-5044-7252); Ana Maria B. Menezes (0000-0002-2996-9427); Marlos R. Domingues (0000-0002-2503-2944); Diego G. Bassani (00000001-6704-3820).

\section{Acknowledgments}

We thank the participants of the 2015 Pelotas (Brazil) birth cohort, other researchers and support staff. We wish to acknowledge the contribution of the Pelotas hospitals [São Francisco de Paula University Hospital of the Catholic University of Pelotas (UCPel); University Support Foundation Hospital (FAU) of the Federal University of Pelotas (UFPel); Santa Casa and Miguel Piltcher hospitals] and the Municipal Secretariat of Health, which allowed us access to live birth and deaths declarations, as well as Wellcome Trust, Brazilian National Research Council (CNPq), and Rio Grande do Sul State Research Foundation (FAPERGS). 


\section{References}

1. World Health Organization. Infant mortality: situations and trends. http://www.who.int/ gho/child_health/mortality/neonatal_infant_ text/en/ (accessed on 14/Oct/2017).

2. Intituto Brasileiro de Geografia e Estatística. Brasil em síntese. Taxas de mortalidade infantil. http://brasilemsintese.ibge.gov.br/popu lacao/taxas-de-mortalidade-infantil.html (accessed on 04/Jan/2019).

3. Berquó ES, Cavenaghi SM. Notas sobre os diferenciais educacionais e econômicos da fecundidade no Brasil. Rev Bras Estud Popul 2014; 31:471-82.

4. Leal MC, Szwarcwald CL, Almeida PVB, Aquino EML, Barreto ML, Barros F, et al. Saúde reprodutiva, materna, neonatal e infantil nos 30 anos do Sistema Único de Saúde (SUS). Ciênc Saúde Colet 2018; 23:1915-28.

5. França EB, Lansky S, Rego MAS, Malta DC, França JS, Teixeira R. Principais causas da mortalidade na infância no Brasil, em 1990 e 2015: estimativas do estudo de Carga Global de Doença. Rev Bras Epidemiol 2017; 20 Suppl 1:46-60.

6. Malta DC, Sardinha L, Moura L, Lansky S, Leal MC, Szwarcwald CL, et al. Atualização da lista de causas evitáveis por intervenções do Sistema Única de Saúde. Epidemiol Serv Saúde 2010; 19:173-6.

7. Organização Pan-Americana da Saúde. Indicadores básicos para a saúde no Brasil: conceitos e aplicações. 2a Ed. Brasília: Organização Pan-Americana de Saúde; 2008.

8. Noronha GA, Torres TG, Kale PL. Análise da sobrevida infantil segundo características maternas, da gestação, do parto e do recém nascido na coorte de nascimento de 2005 no município do Rio de Janeiro-RJ, Brasil. Epidemiol Serv Saúde 2012; 21:419-30.

9. Pinheiro CEA, Peres MA, d'Orsi E. Aumento na sobrevida de crianças de grupos de peso baixo ao nascer em Santa Catarina. Rev Saúde Pública 2010; 44:776-84.

10. Almeida WS, Szwarcwald CL. Adequação das informações de mortalidade e correção dos óbitos informados a partir de pesquisa de busca ativa. Ciênc Saúde Colet 2017; 22:3193203.

11. Mello-Jorge MHP, Laurenti R, Gotlieb SLD. Análise da qualidade das estatísticas vitais brasileiras: a experiência de implantação do SIM e do SINASC. Ciênc Saúde Colet 2007; 12:643-54.

12. Ferreira JSA, Vilela MBR, Aragão PS, Oliveira RA, Tiné RF. Avaliação da qualidade da informação: linkage entre SIM e SINASC em Jaboatão dos Guararapes (PE). Ciênc Saúde Colet 2011; 16 Suppl 1:1241-6.

13. Silva LP, Moreira CMM, Amorim MHC, Castro DS, Zandonade E. Avaliação da qualidade dos dados do Sistema de Informações sobre Nascidos Vivos e do Sistema de Informações sobre Mortalidade no período neonatal, Espírito Santo, Brasil, de 2007 a 2009. Ciênc Saúde Colet 2014; 19:2011-20.
14. Rossetto EV, Luna EJA. Relacionamento entre bases de dados para vigilância da pandemia de influenza A(H1N1)pdm09, Brasil, 2009-2010. Cad Saúde Pública 2016; 32:e00014115.

15. Fundo das Nações Unidas para a Infância. Consultoria: pesquisa para estimar a prevalência de nascimentos pré-termo no Brasil e explorar possíveis causas. Brasília: Fundo das Nações Unidas para a Infância; 2013.

16. Hallal PC, Bertoldi AD, Domingues MR, Silveira MF, Demarco FF, Silva ICM, et al. Cohort Profile: the 2015 Pelotas (Brazil) Birth Cohort Study. Int J Epidemiol 2017; 47:1048-h.

17. Horta BL, Gigante DP, Gonçalves H, Motta JVS, Loret de Mola C, Oliveira IO, et al. Cohort Profile update: the 1982 Pelotas (Brazil) Birth Cohort Study. Int J Epidemiol 2015; 44:441-e.

18. Gonçalves H, Assunção MCF, Wehrmeister FC, Oliveira IO, Barros FC, Victora CG, et al. Cohort profile update: the 1993 Pelotas (Brazil) Birth Cohort follow-up visits in adolescence. Int J Epidemiol 2014; 43:1082-8.

19. Santos IS, Barros AJD, Matijasevich A, Zanini $\mathrm{R}$, Cesar MAC, Camargo-Figuera FA, et al. Cohort profile update: 2004 Pelotas (Brazil) Birth Cohort Study. Body composition, mental health and genetic assessment at the 6 years follow-up. Int J Epidemiol 2014; 43:1437-a-f.

20. American Academy of Pediatrics Committee on Fetus and Newborn; American College of Obstetricians and Gynecologists Committee on Obstetric Practice. The Apgar score. Pediatrics 2015; 136:819-22.

21. Cnattingius S, Norman M, Granath F, Petersson G, Stephansson O, Frisell T. Apgar score components at 5 minutes: risks and prediction of neonatal mortality. Paediatr Perinat Epidemiol 2017; 31:328-37.

22. Secretaria de Atenção à Saúde, Secretaria de Vigilância em Saúde, Ministério da Saúde. Manual de vigilância do óbito infantil e fetal e do Comitê de Prevenção do Óbito Infantil e Fetal. 2a Ed. Brasília: Ministério da Saúde; 2009.

23. Lawn JE, Blencowe H, Pattinson R, Cousens S, Kumar R, Ibiebele I, et al. Stillbirths: where? When? Why? How to make the data count? Lancet 2011; 377:1448-63.

24. Barbeiro FMS, Fonseca SC, Tauffer MG, Ferreira MSS, Silva FP, Ventura PM, et al. Fetal deaths in Brazil: a systematic review. Rev Saúde Pública 2015; 49:22.

25. Froen JF, Cacciatore J, McClure EM, Kuti O, Jokio AH, Islam M, et al. Stillbirths: why they matter. Lancet 2011; 377:1353-66.

26. World Health Organization. WHO recommendations on antenatal care for a positive pregnancy experience. Geneva: World Health Organization; 2016.

27. Santos IS, Matijasevich A, Barros AJD, Albernaz EP, Domingues MR, Valle NCJ, et al. Avoidable deaths in the first four years of life among children in the 2004 Pelotas (Brazil) birth cohort study. Cad Saúde Pública 2011; 27 Suppl 2:S185-97. 
28. Kropiwiec MV, Franco SC, Amaral AR. Fatores associados à mortalidade infantil em município com índice de desenvolvimento humano elevado. Rev Paul Pediatr 2017; 35:391-8.

29. Wondemagegn AT, Alebel A, Tesema C, Abie W. The effect of antenatal care follow-up on neonatal health outcomes: a systematic review and meta-analysis. Public Health Rev 2018; 39:33.

30. United Nations Children's Fund. Antenatal care. https://data.unicef.org/topic/mater nal-health/antenatal-care/ (accessed on 04/ Jun/2017).

31. Secretaria Executiva, Ministério da Saúde. Programa de Humanização do Parto: humanização no pré-natal e nascimento. Brasília: Ministério da Saúde; 2002.

32. Sania A, Smith ER, Manji K, Duggan C, Masanja $\mathrm{H}$, Kisenge R, et al. Neonatal and infant mortality risk associated with preterm and small for gestational age births in Tanzania: individual level pooled analysis using the intergrowth standard. J Pediatr 2018; 192:6672.e.4.

33. Schoeps D, Almeida MF, Alencar GP, França Jr. I, Novaes HMD, Siqueira AAF, et al. Fatores de risco para mortalidade neonatal precoce. Rev Saúde Pública 2007; 41:1013-22.

34. Kilsztajn S, Rossbach A, Carmo MSN, Sugahara GTL. Assistência pré-natal, baixo peso e prematuridade no Estado de São Paulo, 2000. Rev Saúde Pública 2003; 37:303-10.
35. Li F, Wu T, Lei X, Zhang H, Mao M, Zhang J. The Apgar score and infant mortality. PLoS One 2013; 8:e69072.

36. Iliodromiti S, Mackay DF, Smith GCS, Pell JP, Nelson SM. Apgar score and the risk of causespecific infant mortality: a population-based cohort study. Lancet 2014; 384:1749-55.

37. Tomasi E, Fernandes PAA, Fischer T, Fernando CVS, Silveira DS, Thumé E, et al. Qualidade da atenção pré-natal na rede básica de saúde do Brasil: indicadores e desigualdades sociais. Cad Saúde Pública 2017; 33:e00195815.

39. Victora CG, Aquino EM, Carmo Leal M, Monteiro CA, Barros FC, Szwarcwald CL. Maternal and child health in Brazil: progress and challenges. Lancet 2011; 377:1863-76.

39. Gonçalves CV, Cesar JA, Mendoza-Sassi RA. Qualidade e eqüidade na assistência à gestante: um estudo de base populacional no Sul do Brasil. Cad Saúde Pública 2009; 25:2507-16.

40. Victora CG, Matijasevich A, Silveira M, Santos I, Barros AJ, Barros FC. Socio-economic and ethnic group inequities in antenatal care quality in the public and private sector in Brazil. Health Policy Plan 2010; 25:253-61. 


\section{Resumo}

O estudo teve como objetivo descrever a mortalidade fetal, neonatal e pós-neonatal e fatores associados em participantes da coorte de nascimentos de Pelotas, Brasil, de 2015. O sub-estudo sobre mortalidade infantil acompanhou todos os óbitos no primeiro ano de vida. Foram coletados os dados sobre natimortos (com peso ao nascer $\geq 500 \mathrm{~g}$ e/ou idade gestacional $\geq 20$ semanas), óbitos neonatais (<28 dias de vida) e óbitos pós-neonatais (entre 28 dias e o final do primeiro ano de vida). Foram realizadas análises descritivas com o teste de qui-quadrado de Pearson e regressão logística multinominal para estimar o risco de morte fetal, neonatal e pós-neonatal, comparado com as crianças vivas na coorte (grupo de referência). Foram coletados os dados de 4.329 nascimentos elegíveis, dos quais 54 natimortos. Dos 4.275 nascidos vivos elegíveis, 59 faleceram no primeiro ano de vida. A análise mostrou uma associação entre morte fetal, neonatal e pós-neonatal $(O R=15,60,7,63$ e 5,51, respectivamente) e menos de seis consultas de prénatal. Quando comparados aos nascidos vivos, os natimortos apresentaram maior probabilidade de ter mãe não-branca, e o óbito neonatal mostrou probabilidade 14,09 vezes maior de ocorrer com prematuridade (idade gestacional < 37 semanas). Crianças nascidas por cesariana mostraram probabilidade 3,71 vezes maior de óbito pós-neonatal. Além disso, os óbitos neonatais mostraram probabilidade 102,37 maior de Apgar baixo no quinto minuto. Os achados mostram a necessidade de intervenções precoces durante a gravidez para poder garantir uma assistência pré-natal adequada.

Mortalidade Infantil; Estudos de Coortes; Fatores de Risco

\section{Resumen}

El objetivo del presente estudio fue describir la mortalidad fetal, neonatal y postneonatal, así como sus factores asociados, en participantes de una cohorte de nacimientos en Pelotas, Brasil, durante 2015. La mortalidad infantil se siguió mediante un sub-estudio de todas las muertes durante el primer año de vida. Se recogieron datos de muertes intrauterinas fetales (peso al nacer $\geq 500 \mathrm{~g} y / \mathrm{o} \mathrm{edad}$ gestacional $\geq 20$ semanas), muertes neonatales (< 28 dias de vida), $y$ muertes postneonatales (desde los 28 dias hasta el primer año de vida). Se usaron análisis descriptivos usando el test chi-cuadrado de Pearson y se realizó una regresión logística multinomial para estimar el riesgo de las muertes fetales, neonatales y postneonatales, comparadas con los niños vivos en la cohorte (grupo de referencia). Se recogieron datos de 4.329 nacimientos elegibles de los que 54 murieron durante el periodo fetal. De los 4.275 nacimientos vivos elegibles, 59 murieron durante el primer año de vida. Se estableció una asociación entre las muertes fetales, neonatales $y$ postneonatales $(O R=15,60 ; 7,63$ y 5,51, respectivamente) y contar con menos de seis consultas prenatales. Cuando se comparan con los niños vivos, las muertes fetales tenían una probabilidad mayor si contaban con una madre que no fuera blanca, además, habia más de 14,09 veces más probabilidades de que se produjeran con una edad gestacional pretérmino (< 37 semanas). Cuando lo comparamos con los niños vivos, los niños que nacieron en la sección de partos por cesárea tuvieron una oportunidad 3,71 superior de muerte postneonatal. Asimismo, las muertes neonatales fueron 102,37 veces más propensas de tener un bajo Apgar en el quinto minuto tras el nacimiento. Estos resultados mostraron la necesidad de intervenciones tempranas durante el embarazo, asegurando el acceso a un cuidado prenatal adecuado.

Mortalidad Infantil; Estudios de Cohortes; Factores de Riesgo
Submitted on 11/Apr/2018

Final version resubmitted on 28/Jan/2019 Approved on 06/Feb/2019 